\title{
Towards Modeling of Information Processing Within Business-Processes of Service-Providing Organizations
}

\author{
Sergey V. Kovalchuk ${ }^{1(\bowtie)}$, Anastasia A. Funkner ${ }^{1}$, Ksenia Y. Balabaeva ${ }^{1}$, \\ Ilya V. Derevitskii ${ }^{1}$, Vladimir V. Fonin ${ }^{2}$, and Nikita V. Bukhanov ${ }^{1,3}$ \\ 1 ITMO University, Saint Petersburg, Russia \\ \{kovalchuk, funkner.anastasia,k_balabaeva, ivderevitckii\}@itmo.ru \\ 2 PMT Online, Moscow, Russia \\ fonin@pmtonline.ru \\ 3 LLC "GazpromNeft STC", Saint Petersburg, Russia \\ bukhanov.nv@gazpromneft-ntc.ru
}

\begin{abstract}
The paper presents an ongoing research project aimed towards the development of an approach to modeling of information spreading in complex organizations during providing service to customers and performing domain-specific business processes (BP). The approach is based on the idea of information spreading modeling and includes the view of this process in three main levels (physical, informational, and technical). The multi-layer view enables the integration and automation of obtained models in order to resolve domain-specific tasks of BP understanding, optimization, and extension. Two domain-specific case studies from healthcare and HR-management were considered within the approach proposing a way to resolve existing problems.
\end{abstract}

Keywords: Business process $\cdot$ Information processing $\cdot$ Information system $\cdot$ Complex network

\section{Introduction}

The idea of information processing as a paradigm for a description of complex systems attracts the recent attention of scientific society in various applications ranging from a description of complex physical systems as a source of information to analyzing mutual information in complex social systems [13]. One of the interesting areas with a specific multi-layer view to information processing is the information process in a large organization that involves social, informational, organizational, financial, and other types of resources interconnected within a set of business processes (BP). The most interesting class of such systems is an organization that provides a service to a customer. Taking a broad view of the idea of service, it could cover healthcare, education, social service, legal regulation, and many other systems that involve human agents on both sides (customer and 
provider). One can consider many such systems on different levels, each of which has its complexity and own ways of information processing interpretation. This may include a) information storing and processing in (often distributed) information systems like corporate information systems or domain-specific information systems (like medical information systems, MIS) [5]; b) explicit and implicit (performed without direct regulations and rules) BP implementation in a complex domain-specific environment of an organization [11]; c) complex network of agents and resources of different types and roles as a medium for information and knowledge exchange [15]. Within our ongoing study, we are aimed at the development of a holistic approach for modeling and optimization of existing BPs in such multi-level complex systems by considering it through the lens of information processing.

\section{Related Works}

The proposed approach has a certain level of multidisciplinarity, but two key groups of related should be mentioned due to high maturity. This includes BP modeling and information systems/processes management.

Modeling of business processes is a mature area with a well-grounded theoretical and technological background. One of the important tools here is process mining [1] adopted to multiple areas and provided the foundation for modeling and simulation of BPs [16]. Nevertheless, the area has multiple open issues, including management of complex multi-aspect process modeling [10], complexity $[4,8]$, and interpretability [11] of BP models, various uncertainty $[8,12]$ and performance [9] assessing and management, etc.

The complexity of information processing within an information system attracts certain attention of researchers and practitioners in the area of business and IT management. One of the directions developed in this area is business - IT alignment, which hires various concepts to bring systematization into the management procedures. The approaches include conceptual joining of businessprocess management and IT management [14], co-evolutionary approaches [2], complex adaptive systems [17], etc. Still, the investigations in this area are mainly focused on planning and management from strategic, operational, social points of view.

Although the mentioned areas are well-developed high complexity of considered processes, existing open issues, and multiple levels of consideration in BPs within the presented problem bring certain limitations to the direct application of existing solutions.

\section{Basic Concepts}

We are focused on a specific class of systems which mainly include organizations (or department within an organization) providing a service to a customer (or to other departments). One can identify the following characteristics of such a system: 


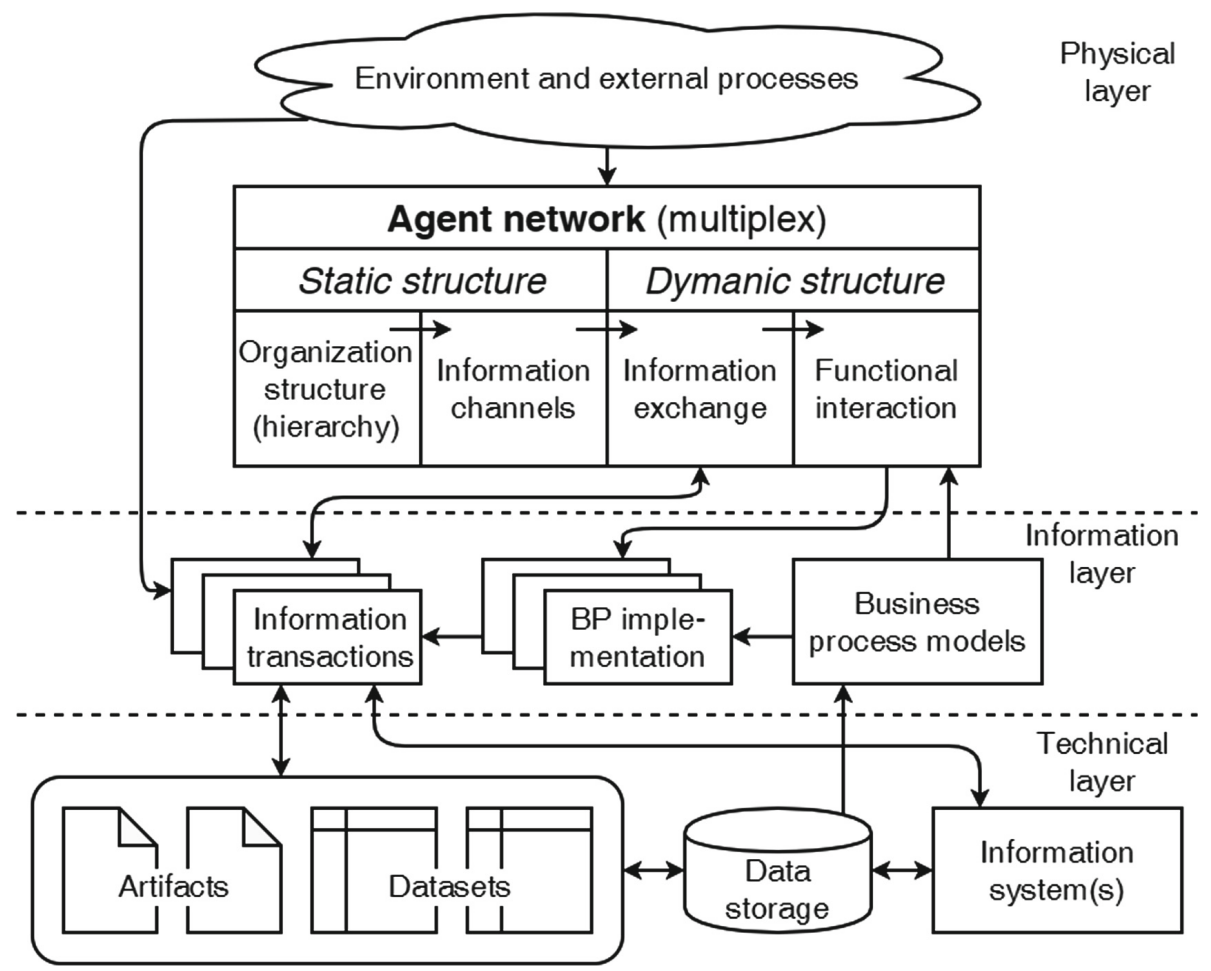

Fig. 1. Information processing in a complex organizational structure.

1. Social interaction within the business processes which include human actors as a subject of BPs and as a target of them.

2. Multi-level, multi-purpose interaction between the agents within the context of BP implementation (including long-distance interaction).

3. High uncertainty of internal or external (within the environment) processes that define or/and influence BPs.

4. Multiple levels of regulation usually include official rules, formal and informal recommendations, common practices etc.

5. Individual strategies, experience, goals, and behavior models of the actors.

6. Diverse, limited, weekly structured data are reflecting the ongoing BP implementation, which commonly includes sparse observations, human-readable artifacts (documents, instructions, messages, etc.) stored in various information systems (including enterprise information systems, EIS).

As a result, such an information processing system obtains characteristics of complex systems [3]. Within our study, we consider such a system as a multilayer structure with a focus on information processing (see Fig. 1). This structure includes three main layers reflecting different points of view to the information processing. 
The physical layer of such a system describes agents within a system. Usually, on the highest level, one can consider the system as a network of agents (in terms of organization, this could include both employees and customers) and the environment where the system resides. The network has a static structure usually defined by a predefined organizational hierarchy and available information channels and dynamic structure characterized by atomic interaction between agents during information exchange and functional co-operating.

The information layer more formally describes the basic objects describing the structure of processes during the service providing inside the system. BP and its particular implementations are based on BPM semantics, which is common to describe processes inside the organization. On the other hand, a set of information transactions may be considered as an atomic object proceeded within a system during BP implementation.

The technical layer includes ways of aggregation, storing, and processing the appearing artifacts (documents, data sets, etc.) within the existing information systems ranging from general-purpose data storage to domain-specific information systems. This layer forms a technological backbone to collect life evidence of the system operating in a common way while providing a service.

In a particular system, different layers may be implemented in various ways. Within our approach, we are aimed towards building a way to reconstruct and estimate information processing in the physical layer through the unification of data in the technical layer and reconstruction and controlling BPs and information transactions in the information layer. Considering technologies for the transition from two bottom layers enable a better understanding of a complex system in the physical layer and optimization of its functional characteristics through the explicit modification of available business-processes and the introduction of new elements in EIS (e.g., in the form of decision support systems). Interconnection of all three layers opens a way to automate BP optimization in a continuous way adapting to the changing of the BPs, their effectiveness (due to the environment changing), or goal criteria defined as a target for organization improvement.

\section{Case Studies}

Currently, we consider several domains that may be mapped onto the proposed idea, including healthcare, human resource management, education, legal regulation, etc. Each of the areas has its own agent roles, structure, and nature of BPs, architecture, and functional characteristics of information systems, sources of uncertainty and process variation, behavioral models, and strategies of agents. Within the following sub-sections, we provide a preliminary analysis of several cases form existing projects in the domains of healthcare and HR management.

\subsection{Distributed Medical Information System}

Remote monitoring of chronic patients is an important task aimed to reveal the peculiarities of chronic disease development (which is usually slowly developed 
and weakly observed process) and treatment effectiveness. We participate in a project performed by PMT Online for developing of a country-scale home monitoring system for chronic disease patients [6]. One of the experimental setup developed within the project was home monitoring of arterial hypertension patients, which perform self-measurements of blood pressure, and the data was transferred to the system via GSM network. The setting was deployed in 83 hospitals in 19 regions of Russia. During January 2019, it collected measurements for about $7 \mathrm{k}$ AH patients treated by 433 physicians (with an average of 16 patients per physician) with about 9 core controlling operators. The agents form a network shown in Fig. 2a, which can be considered as information transfer channels. The system (see the architecture illustrated in Fig. 2b) filter the measurement events according to the rule-based procedure into several categories depending on the urgency of physician intervention (initial, with three levels: green, yellow, and red). One of the important questions was how we could tune the filtering rules to reduce the load of the system agents involved in the event processing (operators, experts, physicians) as even in the basic experimental setting, the system generates about 70k events per month? A key requirement here is keeping false-negative triggering as low as possible and optimize false-positive triggering at the same time. A way for tuning the procedure is updating the triggering rules (see Trigger \#1 and Trigger \#2 in Fig. 2b), which controls information transfer from agent to agent.

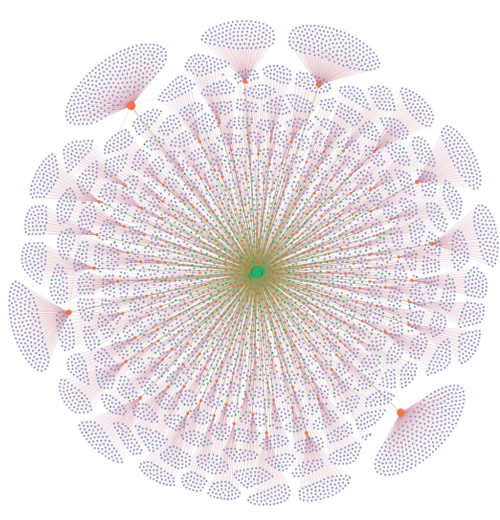

(a) AH monitoring network

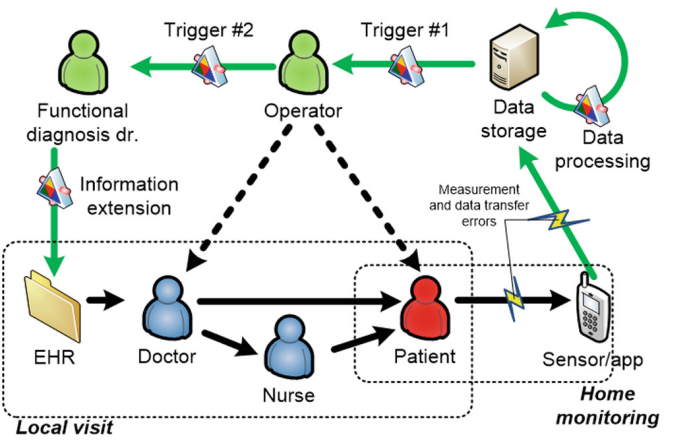

(b) Information loop

Fig. 2. Information processing in a complex organizational structure.

From the point of view of the proposed approach, the physical layer includes a network of patients, physicians, experts, and operators interconnected with different information channels, including MIS-based data transfer, phone calls, messaging, etc. The BP for data processing is quite straight-forward (see Fig. 2b) but is subject to control and optimization. The technical layer includes distributed MIS integrated local MISes in hospitals. Having in mind the idea of information 
transfer, we are developing the simulation version of such a system, including a patient's blood pressure dynamics [7] and controllable state prediction [6] to discover the best triggering rules to reduce redundant information flow.

\subsection{Implicit Business Processes in Company Network}

A large company usually implements a multitude of BPs during its regular activity. This includes explicit BPs defined in the standards, rule books, recommendations, etc. and implicit BPs defined by best practices, common sense, basic organization principles, etc. Within such a complex structural and functional environment, single employee activity may vary significantly. A research question raised within a project performed with GazpromNeft STC was how we could describe and understand a complex behavior of an employee having diverse artifacts within a "digital trail" of his/her activity within a common business process. The goal was to develop a DSS for HR management, taking into account a multitude of factors (formal, functional, informal, behavioral, etc.) influencing effectiveness, skills, (self-)assessment of an employee. Could we identifying advance under- and over-qualified persons, persons under the stress conditions, persons to be promoted or fired, etc.

One of the original practices of the company was a self-defined description of activities within existing BPs. Analyzing a set of 869 such semis-structured descriptions we've identified an organizational structure of the company (Fig. 3a, colors stays for different departments' areas) as well as the closeness of basic BPs within a company (Fig. 3b, 21 clusters of BPs were identified in the network and interpreted with domain-specific meaning, shown in colors). At the same time, an important reflection of information transfer and BP implementation was the implementation of the task of filling the self-defined description. We've discovered a major portion of content sharing in such documents (see Fig. 3c and d), which reflects the dynamic (having a timestamp for the provided documents) and functional interaction between the employees on company scale (including peer-to-peer sharing, providing samples, etc.). All three networks have different topology. Also, depending on BPs, functional, and structural interconnection, various patterns of information spreading could be discovered. E.g., Fig. 3e shows the result of simulation of simple random iterating through the graph of text sharing considering with or without filtering exact copying ("all" and "deg+" in the figure correspondingly), which can be treated as a rough estimation of information spreading within the network. Further work in this direction will be aimed towards the identification of information pathways according to the objective and subjective (self-defined) BPs structure. 


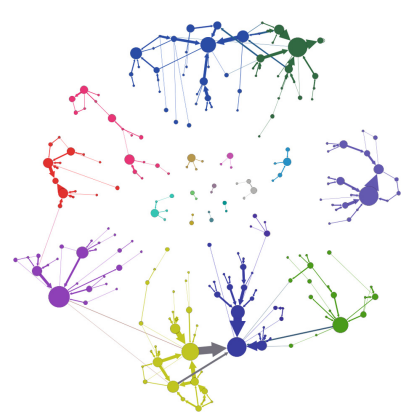

(a) Departments structure

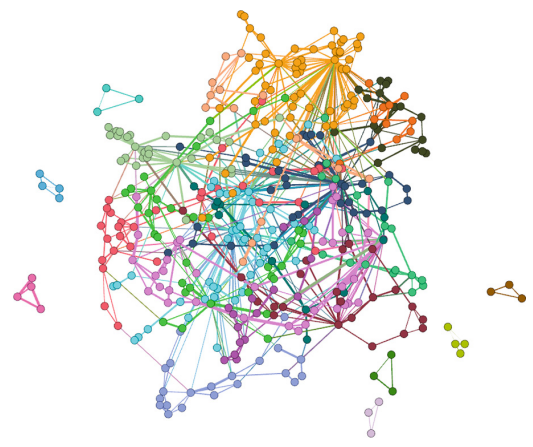

(b) BP interconnection

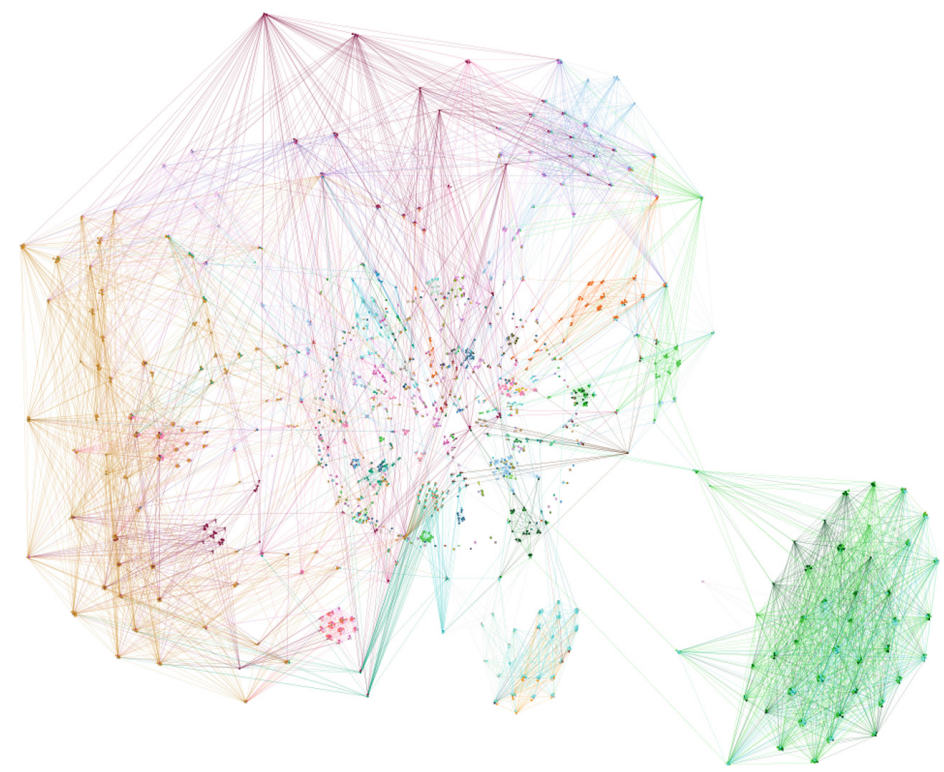

(c) Document sharing network

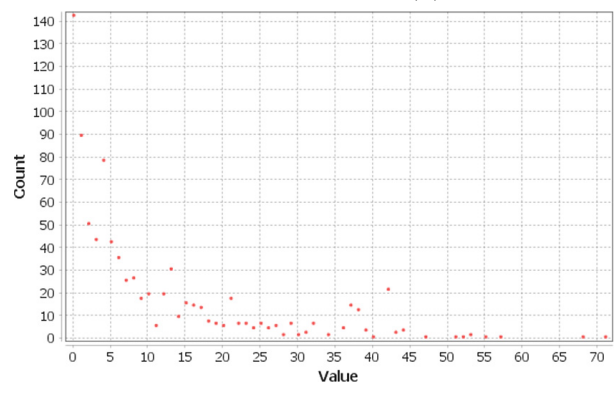

(d) Degree distribution (sharing)

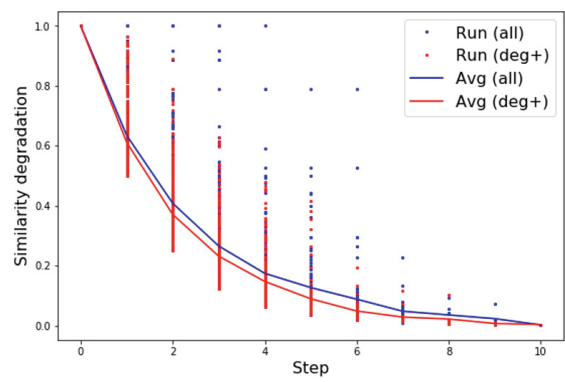

(e) Similarity degradation (sharing)

Fig. 3. Information processing in a complex organizational structure. 


\section{Conclusion and Future Work}

The presented work is currently ongoing. Within the proposed conceptual approach of multi-layered information spreading in the organization, we've considered several domain-specific tasks defining a way to understand emergent phenomena within the information spreading networks. Having defined BPs as a basic structure for dynamic processing of information, there still is a certain variation in $\mathrm{BP}$ implementation. The selected examples include case studies in healthcare and HR management areas.

Further research directions include three main directions. First, the proposed concept of multi-layer information spreading with $\mathrm{BP}$ as a core objective will be defined more formally and without a detailed explanation. Second, the deeper elaboration of proposed case studies will be performed to assess the mutual information in the system's elements, information spreading pathways, and finally, a way to resolve the proposed problems. Finally, seeking problems and applications in other domains will be performed.

Acknowledgments. This work was supported by the Ministry of Science and Higher Education of Russian Federation, Goszadanie No. 2019-1339.

\section{References}

1. van der Aalst, W.: Process Mining, 2nd edn. Springer, Heidelberg (2016). https:// doi.org/10.1007/978-3-662-49851-4

2. Amarilli, F., Van Vliet, M., Van Den Hooff, B.: An explanatory study on the coevolutionary mechanisms of business IT alignment. In: Proceedings of the 38th International Conference on Information Systems, Seoul 2017, pp. 1-22 (2018)

3. Boccara, N.: Modeling Complex Systems. Graduate Texts in Physics. Springer, New York (2010). https://doi.org/10.1007/978-1-4419-6562-2

4. Cardoso, J.: Business process control-flow complexity: metric, evaluation, and validation. Int. J. Web Serv. Res. (IJWSR) 5(2), 49-76 (2008)

5. Delone, W.H., McLean, E.R.: The DeLone and McLean model of information systems success: a ten-year update. J. Manag. Inf. Syst. 19(4), 9-30 (2003)

6. Elkhovskaya, L., Kabyshev, M., Funkner, A., Balakhontceva, M., Fonin, V., Kovalchuk, S.: Personalized assistance for patients with chronic diseases through multi-level distributed healthcare process assessment. Stud. Health Technol. Inform. 261, 309-312 (2019)

7. Funkner, A.A., Zvartau, N.E., Kovalchuk, S.V.: Motif identification in vital signs of chronic patients. Procedia Comput. Sci. 156, 105-113 (2019)

8. Jung, J.Y., Chin, C.H., Cardoso, J.: An entropy-based uncertainty measure of process models. Inf. Process. Lett. 111(3), 135-141 (2011)

9. Leemans, M., Van Der Aalst, W.M., Van Den Brand, M.G.: Hierarchical performance analysis for process mining. In: Proceedings of the 2018 International Conference on Software and System Process, pp. 96-105 (2018)

10. Mendling, J., Baesens, B., Bernstein, A., Fellmann, M.: Challenges of smart business process management: an introduction to the special issue. Decis. Support Syst. 100, 1-5 (2017). https://doi.org/10.1016/j.dss.2017.06.009 
11. Mendling, J., Reijers, H.A., Cardoso, J.: What makes process models understandable? In: Alonso, G., Dadam, P., Rosemann, M. (eds.) BPM 2007. LNCS, vol. 4714, pp. 48-63. Springer, Heidelberg (2007). https://doi.org/10.1007/978-3-540-751830_4

12. Pegoraro, M., Uysal, M.S., van der Aalst, W.M.: Efficient construction of behavior graphs for uncertain event data. arXiv preprint arXiv:2002.08225 (2020)

13. Quax, R., Apolloni, A., Sloot, P.M.A.: Towards understanding the behavior of physical systems using information theory. Eur. Phys. J. Spec. Top. 222(6), 13891401 (2013)

14. Rahimi, F., Møller, C., Hvam, L.: Business process management and IT management: the missing integration. Int. J. Inf. Manag. 36(1), 142-154 (2016). https:// doi.org/10.1016/j.ijinfomgt.2015.10.004

15. Reagans, R., McEvily, B.: Network structure and knowledge transfer: the effects of cohesion and range. Adm. Sci. Q. 48(2), 240 (2003)

16. Rozinat, A., Wynn, M., van der Aalst, W., ter Hofstede, A., Fidge, C.: Workflow simulation for operational decision support. Data Knowl. Eng. 68(9), 834-850 (2009). https://doi.org/10.1016/j.datak.2009.02.014

17. Schilling, R.D., Kazem Haki, M., Beese, J., Aier, S., Winter, R.: Revisiting the impact of information systems architecture complexity: a complex adaptive systems perspective. In: Proceedings of the 38th International Conference on Information Systems, Seoul 2017, pp. 1-18 (2018) 